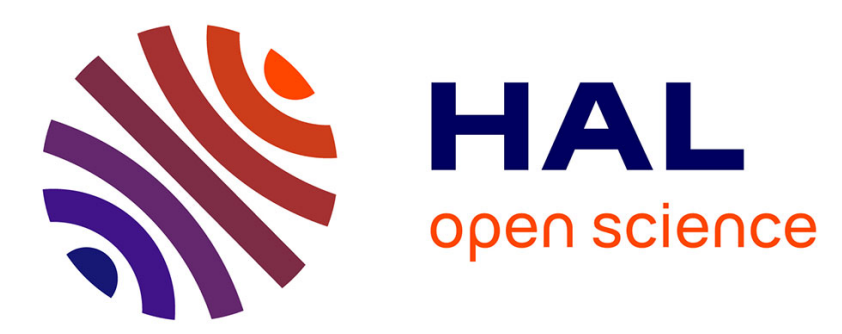

\title{
Any discontinuous PWA function is optimal solution to a parametric linear programming problem
}

Ngoc Anh Nguyen, Sorin Olaru, Pedro Rodriguez-Ayerbe

\section{To cite this version:}

Ngoc Anh Nguyen, Sorin Olaru, Pedro Rodriguez-Ayerbe. Any discontinuous PWA function is optimal solution to a parametric linear programming problem. 54th IEEE Conference on Decision and Control (CDC), Dec 2015, Osaka, Japan. pp.5926-5931, 10.1109/CDC.2015.7403151 . hal-01367123

\section{HAL Id: hal-01367123 \\ https://hal-centralesupelec.archives-ouvertes.fr/hal-01367123}

Submitted on 15 Sep 2016

HAL is a multi-disciplinary open access archive for the deposit and dissemination of scientific research documents, whether they are published or not. The documents may come from teaching and research institutions in France or abroad, or from public or private research centers.
L'archive ouverte pluridisciplinaire HAL, est destinée au dépôt et à la diffusion de documents scientifiques de niveau recherche, publiés ou non, émanant des établissements d'enseignement et de recherche français ou étrangers, des laboratoires publics ou privés. 


\title{
Any discontinuous PWA function is optimal solution to a parametric linear programming problem
}

\author{
N. A. Nguyen ${ }^{1}$, S. Olaru ${ }^{1}$, P. Rodriguez-Ayerbe ${ }^{1}$
}

\begin{abstract}
Recent studies have investigated the continuous functions in terms of inverse optimality. The continuity is a primordial structural property which is exploited in order to link a given piecewise affine (PWA) function to an optimization problem. The aim of this work is to deepen the study of the PWA functions in the inverse optimality context and specifically deal with the presence of discontinuities. First, it will be shown that a solution to the inverse optimality problem exists via a constructive argument. The loss of continuity will have an implication on the structure of the optimization problem which, albeit convex, turns to have a set-valued optimal solution. As a consequence, the original PWA function will represent an optimal solution but the uniqueness is lost. From the numerical point of view, we introduce an algorithm to construct an optimization problem that admits a given discontinuous PWA function as an optimal solution. This construction is shown to rely on convex liftings. A numerical example is considered to illustrate the proposal.
\end{abstract}

\section{INTRODUCTION}

Piecewise affine (PWA) functions have been shown to provide relevance in control design through many studies e.g. [3], [8], [23], [9], [19], [2], [18], [20], [4]. It is known that from the practical point of view, PWA control law has two major limitations in terms of implementation once the number of regions in the state space becomes large:

- it requires substantial memory to store the state space partition and the associated control law gains,

- the point-location problem, determining to which region the current state belongs, becomes more expensive.

Due to the above limitations, many studies have been focused on the complexity reduction of PWA control laws e.g. [11], [10], [12], [6]. Recently, inverse parametric convex programming problem has received an increasing attention in control community as a promising approach to realize this purpose. It is shown in [1] that any continuous nonlinear function is obtainable through a parametric convex programming problem without any hint for the construction of such an inverse optimality problem. Subsequently, some recent studies focus on the recovery of continuous PWA functions defined over polyhedral partitions via the decomposition into the difference of two convex functions as in [7], or via convex liftings as in [16], [17]. As emphasized, all these studies focus on the continuous PWA functions, and the present study aims to compensate the lack of interest for the discontinuous PWA functions by investigating the inverse

N. A. Nguyen, S. Olaru, P. Rodriguez-Ayerbe are with the Laboratory of Signals and Systems (L2S, UMR CNRS 8506), CentraleSupélec-CNRSUniversité Paris Sud, 3 rue Joliot Curie, Plateau de Moulon, 91192, Gif-sur-Yvette, France. Ngocanh.Nguyen, Sorin.Olaru, Pedro.Rodriguez-Ayerbedsupelec.fr optimality. Note also that due to the continuity of the given PWA function, the optimal solution to inverse optimality formulation is unique. However, the uniqueness may no longer be preserved for discontinuous PWA functions. Therefore, finding an optimization problem, whose optimal solution is unique and equivalent to the given discontinuous PWA function, may render this optimization problem nonlinear (non-convex) at least from the point of view of the cost function.

Motivated from the above discussion, in this paper, we restrict our effort to show how to construct a convex optimization problem that admits the given discontinuous PWA function as an optimal solution at the price of non-unicity. Note also that the equivalence between PWA functions is understood in the sense that the boundary between two neighboring regions sharing the same affine function, can be adjusted (subdivided). This optimization problem will be shown to be of parametric linear programming type.

\section{PROBLEM FORMULATION}

\section{A. Generalities and basic notions}

Throughout the paper, $\mathbb{R}, \mathbb{R}_{+}, \mathbb{N}, \mathbb{N}_{>0}$ denote the field of real numbers, the set of non-negative real numbers, the set of nonnegative integers and the positive integer set, respectively. The following index set is also defined, for ease of presentation, with respect to a given $N \in \mathbb{N}_{>0}$ : $\mathcal{I}_{N}=\left\{i \in \mathbb{N}_{>0} \mid i \leq N\right\}$.

Given a finite set $\mathcal{S}:=\left\{s_{1}, \ldots, s_{L}\right\}$, then $\operatorname{Card}(\mathcal{S})$ denotes the cardinal number of $\mathcal{S}$, i.e. $\operatorname{Card}(\mathcal{S})=L \cdot \operatorname{conv}(\mathcal{X})$ denotes the convex hull of $\mathcal{X}$. Moreover, if $\mathcal{R}$ is a set of vectors, by cone $(\mathcal{R})$, we denote the cone which has elements of $\mathcal{R}$ as its extreme rays.

A polyhedron is defined as the intersection of a finite number of halfspaces. A polytope is defined as a bounded polyhedron. Given a full dimensional polyhedron $P \subset \mathbb{R}^{d}$, then $\mathcal{V}(P)$ denotes the set of its vertices, and $\mathcal{R}(P)$ denotes the set of its extreme rays. We use $k$-face to denote a face of dimension $k$ of $P$. A 0 -face is also called a vertex, an 1 -face is alternatively called an edge, a $(d-1)$-face amounts to a facet. Also, $\mathcal{F}(P)$ denotes the set of facets of $P$. Furthermore, by $\operatorname{int}(\mathcal{S})$, we denote the interior of a full dimensional set $\mathcal{S}$. If $\mathbb{S}$ is a vector space, and $\mathcal{S}$ is an arbitrary set, then $\operatorname{Proj}_{\mathbb{S}} \mathcal{S}$ represents the orthogonal projection of the set $\mathcal{S}$ onto the space $\mathbb{S}$. Also, we use $\operatorname{dim}(\mathcal{S})$ to denote the dimension of the affine hull of $\mathcal{S}$.

Given two full dimensional sets $P_{1}, P_{2} \subset \mathbb{R}^{d}$, then the Minkowski sum of these two sets, denoted by $P_{1} \oplus P_{2}$, is 
defined as follows:

$$
P_{1} \oplus P_{2}=\left\{y \in \mathbb{R}^{d} \mid \exists x_{1} \in P_{1}, x_{2} \in P_{2}, y=x_{1}+x_{2}\right\} .
$$

The following definitions represent extensions of the ones introduced in [17], [16], [14].

Definition 1: A collection of $N \in \mathbb{N}_{>0}$ full-dimensional polyhedra $\mathcal{X}_{i} \subset \mathbb{R}^{d}$ is called a polyhedral partition of a polyhedron $\mathcal{X} \subseteq \mathbb{R}^{d}$ if:

1) $\mathcal{X}=\bigcup_{i \in \mathcal{I}_{N}} \mathcal{X}_{i}$

2) $\operatorname{int}\left(\mathcal{X}_{i}\right) \bigcap \operatorname{int}\left(\mathcal{X}_{j}\right)=\emptyset$ with $i \neq j,(i, j) \in \mathcal{I}_{N}^{2}$,

Also, $\left(\mathcal{X}_{i}, \mathcal{X}_{j}\right)$ are called neighbours if $(i, j) \in \mathcal{I}_{N}^{2}, i \neq j$ and $\operatorname{dim}\left(\mathcal{X}_{i} \cap \mathcal{X}_{j}\right)=d-1$.

The notion of cell complex was presented by Grünbaum in [5]. For simplicity, a cell complex can be understood as a polyhedral partition whose facet-to-facet property is fulfilled i.e. any pair of neighboring regions share a common facet.

For ease of presentation, a slight abuse of notation is used in the remainder of this paper: $\mathcal{X}$ denotes simultaneously a polyhedral partition/cell complex of a polyhedron and this polyhedron itself. Its meaning will be clear from the context.

Note that a PWA function $f_{\text {pwa }}(x): \mathbb{R}^{d} \rightarrow \mathbb{R}^{n}$ defined over a polyhedral partition $\mathcal{X}=\bigcup_{i \in \mathcal{I}_{N}} \mathcal{X}_{i} \subseteq \mathbb{R}^{d}$, can be described as follows:

$$
f_{p w a}(x)=h_{i} x+g_{i} \text { for } x \in \mathcal{X}_{i} .
$$

With respect to Definition 1, a PWA function is continuous as long as for any neighboring regions $\left(\mathcal{X}_{i}, \mathcal{X}_{j}\right)$,

$$
h_{i} x+g_{i}=h_{j} x+g_{j}, \forall x \in \mathcal{X}_{i} \cap \mathcal{X}_{j} .
$$

Otherwise, if $f_{\text {pwa }}(x)$ is discontinuous, this discontinuity happens at frontiers between neighboring regions. Thus, such a discontinuous function $f_{p w a}(x)$ can be defined as follows:

$$
f_{p w a}(x)=\left\{\begin{array}{l}
h_{i} x+g_{i} \text { for } x \in \operatorname{int}\left(\mathcal{X}_{i}\right), \\
h_{i} x+g_{i} \text { or } h_{j} x+g_{j} \text { for } x \in \mathcal{X}_{i} \cap \mathcal{X}_{j},
\end{array}\right.
$$

for every pair of neighboring regions $\left(\mathcal{X}_{i}, \mathcal{X}_{j}\right)$. An alternative way to deal with the discontinuities is to allow multiple values in the codomain $\mathbb{R}^{n}$ for a given point $x$. In this case, we are not dealing anymore with a function but a set-valued map.

Definition 2: For a given polyhedral partition $\mathcal{X}=$ $\bigcup_{i \in \mathcal{I}_{N}} \mathcal{X}_{i} \subseteq \mathbb{R}^{d}$, a piecewise affine lifting is described by a real-valued function $z: \mathcal{X} \rightarrow \mathbb{R}$ with:

$$
z(x)=a_{i} x+b_{i} \text { for any } x \in \mathcal{X}_{i},
$$

and $a_{i} \in \mathbb{R}^{d}, b_{i} \in \mathbb{R}, \forall i \in \mathcal{I}_{N}$.

Definition 3: Given a polyhedral partition $\mathcal{X}=\bigcup_{i \in \mathcal{I}_{N}} \mathcal{X}_{i}$ $\subseteq \mathbb{R}^{d}$, a piecewise affine lifting $z(x)=a_{i} x+b_{i} \forall x \in \mathcal{X}_{i}$, is called convex piecewise affine lifting if the following conditions hold true:

- $z(x)$ is continuous over $\mathcal{X}$,

- for each $i \in \mathcal{I}_{N}, z(x)>a_{j} x+b_{j}$ for all $x \in \mathcal{X}_{i} \backslash \mathcal{X}_{j}$ and all $j \neq i, j \in \mathcal{I}_{N}$.

For ease of presentation, a convex piecewise affine lifting is called, throughout the rest of this paper, convex lifting. It is shown in [21], [15] that a polyhedral partition which admits a convex lifting, is a cell complex. Therefore, a convex lifting is always defined over a cell complex instead of a polyhedral partition.

As discussed above, a given polyhedral partition has to fulfill some conditions for the existence of a convex lifting. A summary of these conditions can be found in [21], [17].

Definition 4: A given cell complex $\mathcal{X}=\bigcup_{i \in \mathcal{I}_{N}} \mathcal{X}_{i} \subseteq$ $\mathbb{R}^{d}$ has an affinely equivalent polyhedron if there exists a polyhedron $\tilde{\mathcal{X}} \subset \mathbb{R}^{d+1}$ such that for each $i \in \mathcal{I}_{N}$ :

1) $\exists F_{i} \in \mathcal{F}(\widetilde{\mathcal{X}})$ satisfying: $\operatorname{Proj}_{\mathbb{R}^{d}} F_{i}=\mathcal{X}_{i}$,

2) if $\underline{z}(x)=\min _{z} z$ s.t. $\left[x^{T} z\right]^{T} \in \widetilde{\mathcal{X}}$, then $\left[\begin{array}{c}x \\ z \\ (x)\end{array}\right] \in F_{i}$ for $x \in \mathcal{X}_{i}$.

\section{B. Problem formulation}

As mentioned above, the solutions to inverse parametric linear/quadratic programming problems in [7], [17], [16] are known to only recover continuous PWA functions and to exclude the class of discontinuous PWA functions. This paper concentrates on inverse optimality for the latter class of functions and shows that it can be obtained via convex liftings. This problem can be stated as follows:

\section{Problem statement:}

Let a polyhedral partition of a polyhedron $\mathcal{X}=\bigcup_{i \in \mathcal{I}_{N}} \mathcal{X}_{i} \subseteq$ $\mathbb{R}^{d_{x}}$, and a discontinuous PWA function: $f_{\text {pwa }}(\cdot): \mathcal{X} \rightarrow$ $\mathbb{R}^{d_{u}}$. The goal is to determine $J(x, z, u), H_{x}, H_{u}, H_{z}, K$ such that:

$$
\left\{\begin{aligned}
f_{p w a}(x) & \left.\in \operatorname{Proj}_{\mathbb{R}^{d_{u}}} \arg \min _{[z} u^{T}\right]^{T} \\
\text { s.t: } & \left.H_{x} x+H_{z} z+H_{u} u \leq K, z, u\right),
\end{aligned}\right.
$$

The following assumption is necessary for guaranteeing a well-posed problem.

Assumption 1: Polyhedral partition $\mathcal{X}$ is convexly liftable. Note that Assumption 1 is not restrictive, in view of Theorem IV.2 in [17]. This theorem is recalled here for completeness.

Theorem 2.1: Given a non convexly liftable polyhedral partition $\mathcal{X}=\bigcup_{i \in \mathcal{I}_{N}} \mathcal{X}_{i} \subseteq \mathbb{R}^{d}$, there exists at least one subdivision, preserving the internal boundaries of this partition, such that the new cell complex is convexly liftable.

In virtue of this result, if a given polyhedral partition is not convexly liftable, one can subdivide it into a convexly liftable partition such that the internal boundaries are maintained. This new partition is equivalent to the initial one. Therefore, we can focus on convexly liftable partitions throughout the rest of this paper.

Note that this result holds true not only for polytopic partitions (bounded polyhedral partitions) as shown in [17] but also for polyhedral partitions of a polyhedron. The proof is similar to the one presented therein and is omitted for brevity.

\section{Constructive SOlution}

\section{A. Parametric linear programming}

Constructive solution to inverse optimality problem in this paper is based on convex liftings. It is worth mentioning 
that the algorithm to construct convex liftings, put forward in [17], is not directly applicable for polyhedral partition of polyhedra, since this algorithm is based on the constraints imposed at related vertices. An extension to convex liftings for polyhedral partitions of a polyhedron is referred to [13]. Based on the above necessary condition, we can state the main result of this paper.

We consider a cell complex of a polyhedron $\mathcal{X}=$ $\bigcup_{i \in \mathcal{I}_{N}} \mathcal{X}_{i} \subseteq \mathbb{R}^{d_{x}}$ satisfying Assumption 1 and a possibly discontinuous PWA function $f_{p w a}(\cdot): \mathcal{X} \rightarrow \mathbb{R}^{d_{u}}$ defined as follows:

$$
f_{\text {pwa }}(x)=\left\{\begin{array}{l}
h_{i} x+g_{i} \text { for } x \in \operatorname{int}\left(\mathcal{X}_{i}\right), \\
h_{i} x+g_{i} \text { or } h_{j} x+g_{j} \text { for } x \in \mathcal{X}_{i} \cap \mathcal{X}_{j},
\end{array}\right.
$$

where $\left(\mathcal{X}_{i}, \mathcal{X}_{j}\right)$ are neighbors. We use $\ell(x)$ to denote a convex lifting of this cell complex, i.e.

$$
\ell(x)=a_{i} x+b_{i}, \text { for } x \in \mathcal{X}_{i} .
$$

Also, by $f_{p w a}^{(i)}(x)$, we denote the $i^{\text {th }}$ component of the function $f_{p w a}(x)$ at $x$ for $i \in \mathcal{I}_{d_{u}}$. The following sets are defined:

$$
\begin{aligned}
& V_{x}=\bigcup_{i \in \mathcal{I}_{N}} \mathcal{V}\left(\mathcal{X}_{i}\right), R_{x}=\bigcup_{i \in \mathcal{I}_{N}} \mathcal{R}\left(\mathcal{X}_{i}\right), \\
& \bar{f}_{p w a}^{(i)}(x)=\max _{j \in \mathcal{I}_{N} \mid x \in \mathcal{X}_{j}} h_{j}^{(i)} x+g_{j}^{(i)}, \\
& \underline{f}_{p w a}^{(i)}(x)=\min _{j \in \mathcal{I}_{N} \mid x \in \mathcal{X}_{j}} h_{j}^{(i)} x+g_{j}^{(i)} .
\end{aligned}
$$

Note that $\bar{f}_{p w a}^{(i)}(x)\left(\underline{f}_{p w a}^{(i)}(x)\right)$ is defined as the maximal (minimal) value among the values of the $i^{\text {th }}$ component of the affine functions composing $f_{p w a}(x)$ at $x$, defined over the regions which contain $x$.

$$
\begin{aligned}
& \mathbf{U}(x)=\left\{\left[\begin{array}{c}
u^{(1)}(x) \\
\vdots \\
u^{\left(d_{u}\right)}(x)
\end{array}\right] \mid u^{(i)}(x) \in\left\{\bar{f}_{p w a}^{(i)}(x), \underline{f}_{p w a}^{(i)}(x)\right\}\right\}, \\
& V_{\left[x^{T} z u^{T}\right]^{T}}=\left\{\left[\begin{array}{c}
x \\
\ell(x) \\
u(x)
\end{array}\right] \mid x \in V_{x}, u(x) \in \mathbf{U}(x)\right\}, \\
& R_{\left[x^{T} z u^{T}\right]^{T}}=\left\{\left[\begin{array}{c}
r \\
\widehat{\ell}(r) \\
\widehat{h}(r)
\end{array}\right] \mid \begin{array}{l}
r \in R_{x}, \\
\widehat{\ell}(r)=a_{i} r \\
\widehat{h}(r)=h_{i} r
\end{array} \text { if } r \in \mathcal{R}\left(\mathcal{X}_{i}\right)\right\}, \\
& \Pi_{v}=\operatorname{conv}\left(V_{\left[x^{T} z u^{T}\right]^{T}}\right), \Pi_{r}=\operatorname{cone}\left(R_{\left[x^{T} z u^{T}\right]^{T}}\right), \\
& \Pi=\Pi_{v} \oplus \Pi_{r} .
\end{aligned}
$$

Note that $V_{\left[x^{T} z u^{T}\right]^{T}}\left(R_{\left[x^{T} z u^{T}\right]^{T}}\right)$ represents the set of extended vertices (extreme rays) of the partition $\mathcal{X}$ in the augmented space. Also from the above construction, if for a given $x \in \mathcal{X}$, we have $\underline{f}_{p w a}^{(i)}(x)=\bar{f}_{p w a}^{(i)}(x)$ for all $i \in \mathcal{I}_{d_{u}}$, then the function $f_{p w a}(x)$ is continuous at $x$. In this case, $\operatorname{Card}(\mathbf{U}(x))=1$. The following observation is useful for the next development.
Proposition 3.1: For any $x \in \mathcal{X}$ and $\mathbf{U}(x)$ defined in (6), $\mathbf{U}(x)=\mathcal{V}(\operatorname{conv}(\mathbf{U}(x)))$.

Proof: Suppose $\mathcal{V}(\operatorname{conv}(\mathbf{U}(x))) \subset \mathbf{U}(x)$, then there exists a point $u_{0}(x) \in \mathbf{U}(x)$ for a given $x \in \mathcal{X}$ such that $u_{0}(x)$ can be described via a convex combination of the other points $u(x) \in \mathbf{U}(x), u(x) \neq u_{0}(x)$, then

$$
\begin{aligned}
& u_{0}(x)=\sum_{u(x) \neq u_{0}(x), u(x) \in \mathbf{U}(x)} \alpha(u(x)) u(x), \\
& \alpha(u(x)) \geq 0, \quad \sum_{u(x) \neq u_{0}(x), u(x) \in \mathbf{U}(x)} \alpha(u(x))=1 .
\end{aligned}
$$

We need to find a contradiction. Indeed, $u_{0}^{(1)}(x)$ takes value in the discrete set $\left\{\bar{f}_{p w a}^{(1)}(x), \underline{f}_{p w a}^{(1)}(x)\right\}$, and any of these two values cannot be described by a convex combination of the other one if they are different. Thus, $u_{0}^{(1)}(x)=\sum_{u(x) \neq u_{0}(x), u(x) \in \mathbf{U}(x)} \alpha(u(x)) u^{(1)}(x)$ holds true if $u^{(1)}(x)=u_{0}^{(1)}(x)$, meaning:

$$
\begin{aligned}
& \sum_{u(x) \neq u_{0}(x), u(x) \in \mathbb{U}(x), u^{(1)}(x)=u_{0}^{(1)}(x)} \alpha(u(x))=1, \\
& \alpha(u(x))=0 \text { for } u^{(1)}(x) \neq u_{0}^{(1)}(x) .
\end{aligned}
$$

The same argument, applied for the other components $u_{0}^{(i)}(x), i \in \mathcal{I}_{d_{u}}$, leads to:

$$
\begin{aligned}
& \alpha(u(x))=1, \text { for } u(x)=u_{0}(x), \\
& \alpha(u(x))=0, \text { for } u(x) \neq u_{0}(x) .
\end{aligned}
$$

This inclusion is clearly contradictory with (7).

Proposition 3.2: $V_{\left[x^{T} z u^{T}\right]^{T}}=\mathcal{V}\left(\Pi_{v}\right)$.

Proof: Suppose there exists a point of $V_{\left[x^{T} z u^{T}\right]^{T} \text {, }}$ denoted by $\left[x^{T} \ell(x) u_{0}^{T}(x)\right]^{T}$, which can be described via a convex combination of the other points in this set. Formally, this leads to the following relationship:

$$
\begin{aligned}
{\left[\begin{array}{c}
x \\
\ell(x) \\
u_{0}(x)
\end{array}\right]=} & \left.\sum \begin{array}{c}
v \\
\ell(v) \\
u(v)
\end{array}\right] \neq\left[\begin{array}{c}
x \\
\ell(x) \\
u_{0}(x)
\end{array}\right], \\
& {\left[\begin{array}{c}
v \\
\ell(v) \\
u(v) \\
u(v)
\end{array}\right] \in V_{\left[x^{T} z u^{T}\right]^{T}} }
\end{aligned}
$$

where

$$
\begin{aligned}
& \alpha\left(\left[\begin{array}{c}
v \\
\ell(v) \\
u(v)
\end{array}\right]\right) \geq 0, \\
& \begin{array}{l}
\sum_{\left[\begin{array}{c}
v \\
\ell(v) \\
u(v)
\end{array}\right] \neq\left[\begin{array}{c}
x \\
\ell(x) \\
u u_{0}(x)
\end{array}\right]} \alpha\left(\left[\begin{array}{c}
v \\
\ell(v) \\
u(v)
\end{array}\right]\right)=1 . \\
{\left[\begin{array}{c}
v \\
\ell(v) \\
u(v)
\end{array}\right] \in V_{\left[x^{T} z u^{T}\right]^{T}}}
\end{array}
\end{aligned}
$$

Due to the fact that any $\left[x^{T} \ell(x)\right]^{T}, x \in V_{x}$, cannot be described via a convex combination of the other 
$\left[v^{T} \ell(v)\right]^{T}, v \neq x, v \in V_{x}$, then relation (8) only holds true for $v=x$, leading to $\alpha\left(\left[\begin{array}{c}v \\ \ell(v) \\ u(v)\end{array}\right]\right)=0$ for $v \neq x$. The remaining coefficients only depend on $u(x)$, therefore for ease of presentation, we denote the remaining coefficients by $\alpha(u(x))$ instead of $\alpha\left(\left[\begin{array}{c}x \\ \ell(x) \\ u(x)\end{array}\right]\right)$.

To complete the proof, we need to show that

$$
\begin{aligned}
& u_{0}(x)=\sum_{u(x) \neq u_{0}(x), u(x) \in \mathbf{U}(x)} \alpha(u(x)) u(x), \\
& \alpha(u(x)) \geq 0, \quad \sum_{u(x) \neq u_{0}(x), u(x) \in \mathbf{U}(x)} \alpha(u(x))=1,
\end{aligned}
$$

leads to a contradiction. This end is easily deduced from Proposition 3.1 and the proof is complete.

Based on the above inclusions, the main result of the paper is presented below.

Theorem 3.3: Any possibly discontinuous PWA function (3) defined over a partition of a polyhedron satisfying Assumption 1 , is the image via the orthogonal projection onto $\mathbb{R}^{d_{u}}$ of an optimal solution to the following optimization problem:

$$
\min _{\left[z u^{T}\right]^{T}} z \quad \text { s.t. } \quad\left[x^{T} z u^{T}\right]^{T} \in \Pi,
$$

where $\Pi$ is defined as in (6).

Proof: Given a point $x \in \mathcal{X}_{i}$ in the cell complex $\mathcal{X}$, due to the Minkowski-Weyl theorem (see Corollary 7.1b in [22]), $x$ can be written as a convex combination as follows:

$$
x=\sum_{v \in \mathcal{V}\left(\mathcal{X}_{i}\right)} \alpha(v) v+\sum_{r \in \mathcal{R}\left(\mathcal{X}_{i}\right)} \beta(r) r,
$$

where $\alpha(v), \beta(r) \geq 0, \sum_{v \in \mathcal{V}\left(\mathcal{X}_{i}\right)} \alpha(v)=1$. Due to Assumption 1 , let $\ell(x)$ denote a convex lifting of the cell complex $\mathcal{X}$, i.e. $\ell(x)=a_{i} x+b_{i}$ for $x \in \mathcal{X}_{i}$. For an $x \in \mathcal{X}_{i}$, it follows that:

$$
\begin{aligned}
\ell(x) & =a_{i} x+b_{i} \\
& =\sum_{v \in \mathcal{V}\left(\mathcal{X}_{i}\right)} \alpha(v)\left(a_{i} v+b_{i}\right)+\sum_{r \in \mathcal{R}\left(\mathcal{X}_{i}\right)} \beta(r) a_{i} r .
\end{aligned}
$$

Thus, if we define the following sets:

$$
\begin{aligned}
& V_{\left[x^{T} z\right]^{T}}=\left\{\left[\begin{array}{c}
x \\
\ell(x)
\end{array}\right] \mid x \in V_{x}\right\}, \\
& R_{\left[x^{T} z\right]^{T}}=\left\{\left[\begin{array}{c}
r \\
\widehat{\ell}(r)
\end{array}\right] \mid \begin{array}{l}
r \in R_{x}, \\
\widehat{\ell}(r)=a_{i} r \text { if } r \in \mathcal{R}\left(\mathcal{X}_{i}\right)
\end{array}\right\},
\end{aligned}
$$

then $\Pi_{\left[x^{T} z\right]^{T}}=\operatorname{conv}\left(V_{\left[x^{T} z\right]^{T}}\right) \oplus \operatorname{cone}\left(R_{\left[x^{T} z\right]^{T}}\right)$ is an affinely equivalent polyhedron of the cell complex $\mathcal{X}$.

Following the definition of an affinely equivalent polyhedron, for a region $\mathcal{X}_{i}$ in the given cell complex $\mathcal{X}$, there exists a lower facet of $\Pi_{\left[x^{T} z\right]^{T}}$, denoted by $F_{\left[x^{T} z\right]^{T}}$ such that $\operatorname{Proj}_{\mathbb{R}^{d_{x}}} F_{\left[x^{T} z\right]^{T}}=\mathcal{X}_{i}$ and the optimal solution to the following optimization problem:

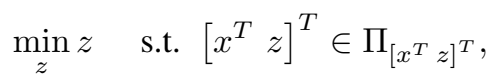

falls in $F_{\left[x^{T} z\right]^{T}}$ for $x \in \mathcal{X}_{i}$. Note however that, due to the construction in (6), every $d_{x}$-face, denoted by $F^{\#}$ and defined as follows:

$$
\begin{aligned}
F^{\#} & =F_{1}^{\#} \oplus F_{2}^{\#}, \\
F_{1}^{\#} & =\operatorname{conv}\left\{\left[v^{T} \ell(v) u^{T}(v)\right]^{T} \mid v \in \mathcal{V}\left(\mathcal{X}_{i}\right)\right\}, \\
F_{2}^{\#} & =\operatorname{cone}\left\{\left[r^{T} a_{i} r\left(h_{i} r\right)^{T}\right]^{T} \mid r \in \mathcal{R}\left(\mathcal{X}_{i}\right)\right\}, \\
u(v) & \in \operatorname{conv}(\mathbf{U}(v)),
\end{aligned}
$$

satisfies: Proj ${\mathbb{R} d_{x}+1}^{\#} F^{\#}=F_{\left[x^{T} z\right]^{T}}$. Also, consider a point $x \in \mathcal{X}_{i}$, optimizer of the optimization problem (10) at $x$, locates on such a $d_{x}$-face $F^{\#}$. Due to this non-uniqueness, we can choose the following $d_{x}$-face, denoted by $\widetilde{F}$, defined as in (11) with $u(v)=h_{i} v+g_{i}$ for $v \in \mathcal{X}_{i}$. Then, for any $x \in \mathcal{X}_{i}$, the optimizer, located on $\widetilde{F}$, satisfies:

$$
\left[\begin{array}{c}
x \\
z^{*}(x) \\
u^{*}(x)
\end{array}\right]=\sum_{v \in \mathcal{V}\left(\mathcal{X}_{i}\right)} \alpha(v)\left[\begin{array}{c}
v \\
\ell(v) \\
h_{i} v+g_{i}
\end{array}\right]+\sum_{r \in \mathcal{R}\left(\mathcal{X}_{i}\right)} \beta(r)\left[\begin{array}{c}
r \\
a_{i} r \\
h_{i} r
\end{array}\right],
$$

meaning $u^{*}(x)=h_{i} x+g_{i}=f_{\text {pwa }}(x)$.

Remark 1: Note that one can easily fix a large enough box in $\mathbb{R}^{d_{u}}$ to bound every $\mathbf{U}(x), \forall x \in V_{x}$. Such a constraint can avoid the computation of $\mathbf{U}(x)$ at each vertex of the cell complex $\mathcal{X}$. However, such a choice ignores the structure of the given PWA function and cannot exploit its continuity property whenever this exists.

Algorithm 1 summarizes the construction presented above.

$\overline{\text { Algorithm } 1 \text { Constructive solution to inverse optimality }}$ problem for discontinuous PWA functions

Input: A discontinuous PWA function (3), i.e. $f_{p w a}(x)$ defined over convexly liftable cell complex $\mathcal{X}$.

Output: $H_{x}, H_{u}, H_{z}, K, J(x, z, u)$.

1: Compute a convex lifting $\ell(x)$ of the cell complex $\mathcal{X}$.

2: Construct a constraint set $\Pi$ as in (6).

3: Define $J(x, z, u)=z$.

4: Solve the following parametric linear programming problem:

$$
\min _{\left[z u^{T}\right]^{T}} z \quad \text { s.t. } \quad\left[x^{T} z u^{T}\right]^{T} \in \Pi .
$$

\section{B. Connections to parametric quadratic programming}

Similar to the above construction, a simple extension from parametric linear programming to a parametric quadratic programming problem is introduced below.

Theorem 3.4: Given a possibly discontinuous PWA function (3) defined over a partition of a polyhedron satisfying Assumption 1, one of its associated convex lifting (4), let $\sigma(x)$ be a function $\sigma(x) \leq \ell(x)$ for $x \in \mathcal{X}$. The given PWA function (3) is the image via the orthogonal projection onto $\mathbb{R}^{d_{u}}$ of an optimal solution to the following parametric quadratic programming problem:

$$
\min _{\left[z u^{T}\right]^{T}}(z-\sigma(x))^{2} \text { s.t. }\left[x^{T} z u^{T}\right]^{T} \in \Pi,
$$


where $\Pi$ is defined as in (6).

Proof: Due to the assumption that $\sigma(x) \leq \ell(x)$ for $x \in \mathcal{X}$, the optimization problem (12) amounts to the minimization of $z$ subject to the same constraints. Therefore, by Theorem 3.3, $f_{p w a}(x)$ is a sub-component of an optimal solution to problem (12). The proof is complete.

To conclude the analysis of piecewise affine functions and their inverse optimality, the following theorem presents the main message of the paper.

Theorem 3.5: Any possibly discontinuous PWA function, defined over a polyhedral partition of a polyhedron, can be equivalently obtained as a selection among the optimal solutions of a parametric linear/quadratic programming problem.

Proof: Let $f_{\text {pwa }}(x)$ denote a given PWA function, defined over a polyhedral partition $\mathcal{X}$. If the partition $\mathcal{X}$ is convexly liftable, Theorems $3.3,3.4$ show in a constructive manner parametric linear/quadratic programming problems for which $f_{p w a}(x)$ is a sub-component of an optimal solution.

Otherwise, if $\mathcal{X}$ is not convexly liftable, as per Theorem 2.1 , one can subdivide $\mathcal{X}$ into a convexly liftable cell complex such that the internal boundaries are maintained. According to this new cell complex, $f_{\text {pwa }}(x)$ is also subdivided into an equivalent PWA function, say $\widetilde{f}_{p w a}(x)$. Note also that this equivalent function $\tilde{f}_{p w a}(x)$ is defined over a convexly liftable partition. Again, according to Theorems 3.3, 3.4, $\widetilde{f}_{\text {pwa }}(x)$ can be obtained via parametric linear/quadratic programming problems.

\section{NUMERICAL EXAMPLE}

Consider a simple discontinuous PWA function $f_{p w a}(x)$, defined over a partition in $\mathbb{R}$ as follows:

$$
f_{p w a}(x)=\left\{\begin{array}{l}
0.8116 x+0.5328 \text { for }-0.4 \leq x<-0.3 \\
0.3507 x+0.9390 \text { for }-0.3 \leq x<-0.2 \\
0.8759 x+0.5502 \text { for }-0.2 \leq x<-0.1 \\
0.6225 x+0.5870 \text { for }-0.1 \leq x<0 \\
0.2077 x+0.3012 \text { for } 0 \leq x<0.1 \\
0.4709 x+0.2305 \text { for } 0.1 \leq x<0.2 \\
0.8443 x+0.1948 \text { for } 0.2 \leq x<0.3 \\
0.2259 x+0.1707 \text { for } 0.3 \leq x \leq 0.4
\end{array}\right.
$$

One can easily check the discontinuity of this function via its values at the vertices of the regions in the parameter space partition (see Fig.1). A convex lifting of this partition denoted by $\ell(x)$, is presented below:

$$
\ell(x)=\left\{\begin{array}{c}
-3.5 x+0.4 \text { for }-0.4 \leq x \leq-0.3 \\
-2.5 x+0.7 \text { for }-0.3 \leq x \leq-0.2 \\
-1.5 x+0.9 \text { for }-0.2 \leq x \leq-0.1 \\
-0.5 x+1 \text { for }-0.1 \leq x \leq 0 \\
0.5 x+1 \text { for } 0 \leq x \leq 0.1 \\
1.5 x+0.9 \text { for } 0.1 \leq x \leq 0.2 \\
2.5 x+0.7 \text { for } 0.2 \leq x \leq 0.3 \\
3.5 x+0.4 \text { for } 0.3 \leq x \leq 0.4
\end{array}\right.
$$

Also, this convex lifting is shown in Fig.2. Following the approach presented previously, an optimization problem that admits the given PWA function as an optimal solution, is presented in (13).

The given discontinuous PWA function is presented in Fig. 1 wherein the line along the horizontal axis describes the parameter space $x$ and the multicolored lines above describe the given PWA function. Also, in the extended space of parameter $x$, the original function $f_{\text {pwa }}(x)$, lifting $\ell(x)$, the green lines in Fig.3 represent the given PWA function. The shaded pink polytope represents a set of constraints $\Pi$ in the inverse optimization problem. Note also that the solid pink lines describe an optimal solution to this optimization problem. Moreover, the images of these lines onto the space of $\left(x, f_{\text {pwa }}(x)\right)$ coincide with the given PWA function. It is also worth emphasizing that for each segment of state space partition, the set of optimal solutions to the above optimization problem represents a facet of the pink polytope $\Pi$, which is orthogonal to the space $(x, z)$. As illustrated in Fig.4, the pink facets represent the set of optimal solutions to the optimization problem (13).

$\min _{\left[z u^{T}\right]^{T}} z \quad$ s.t.
$\left[\begin{array}{cc}-0.8944 & 0 \\ -0.5805 & 0.1893 \\ -0.4462 & 0.3412 \\ -0.5547 & 0 \\ -0.2547 & 0.6733 \\ -0.3714 & 0 \\ -0.2747 & 0 \\ -0.4473 & -0.3356 \\ -0.8944 & 0 \\ -0.8200 & 0.2018 \\ 0.2738 & 0.7989 \\ 0.3139 & 0.8057 \\ -0.6351 & -0.2551 \\ -0.5547 & 0 \\ -0.3714 & 0 \\ -0.4659 & -0.5706 \\ -0.2747 & 0 \\ -0.3217 & -0.8334 \\ -0.1827 & -0.9733 \\ -0.0951 & -0.9955 \\ 0.0455 & -0.9968 \\ 0.8700 & 0.4919\end{array}\right]\left[\begin{array}{l}-0.4472 \\ -0.7920 \\ -0.8273 \\ -0.8321 \\ -0.6941 \\ -0.9285 \\ -0.9615 \\ -0.8290 \\ 0.4472 \\ 0.5356 \\ -0.5355 \\ -0.5022 \\ 0.7291 \\ 0.8321 \\ 0.9285 \\ 0.6763 \\ 0.9615 \\ 0.4494 \\ 0.1390 \\ 0.0045 \\ -0.0659 \\ 0.0325\end{array}\right] x+\left[\begin{array}{l}-0.8944 \\ -0.4693 \\ -0.2459 \\ -0.4992 \\ 0.1406 \\ -0.2600 \\ -0.1099 \\ -0.4799 \\ -0.8944 \\ -0.7015 \\ 0.9156 \\ 0.9763 \\ -0.7119 \\ -0.4992 \\ -0.2600 \\ -0.6378 \\ -0.1099 \\ -0.5727 \\ -0.4759 \\ -0.3766 \\ -0.1520 \\ 1.6815\end{array}\right]$

\section{CONClusions}

This paper presents an inverse optimality result for the class of discontinuous PWA functions. The solution is based on convex liftings and leads to a linear programming type of solution. It is also emphasized that optimal solution to such an optimization problem is no longer unique but a set of solutions containing the given discontinuous PWA function. A numerical example is considered to illustrate these results. The result is mainly theoretical and sheds light 


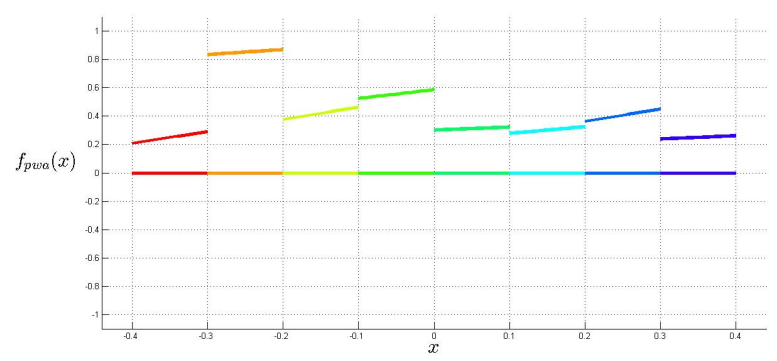

Fig. 1. A discontinuous PWA function defined over a partition in $\mathbb{R}$.

on the difficulties of discontinuous PWA control law design via convex optimization. Basically, it shows that convex lifting can emulate the polyhedral partition and translate to the constraint set of an optimization problem. However, in order to recover the unicity of the optimizer, the cost function needs to embed the discontinuity via a nonlinear function.

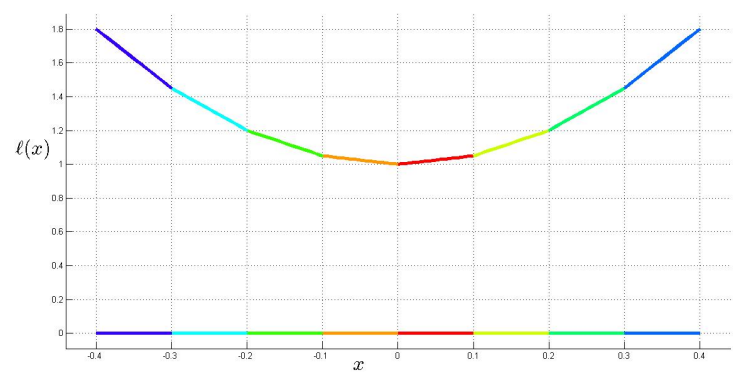

Fig. 2. A convex lifting of the given cell complex.

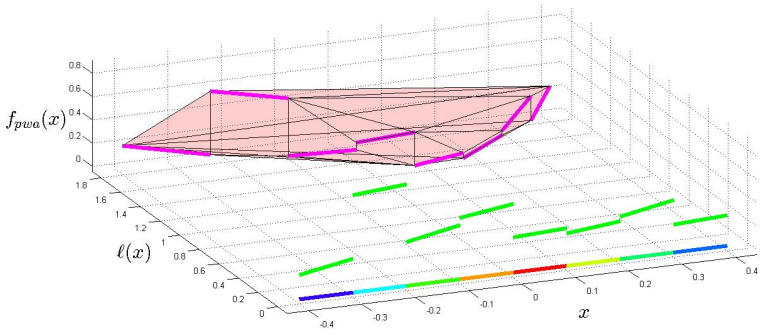

Fig. 3. Graphical illustration of the recovered optimization problem.

\section{REFERENCES}

[1] M. Baes, M. Diehl, and I. Necoara, "Every continuous nonlinear control system can be obtained by parametric convex programming," IEEE tran on Automatic Control, vol. 53, no. 8, pp. 1963-1967, Sept 2008.

[2] A. Bemporad and C. Filippi, "An algorithm for approximate multiparametric convex programming," Computational optimization and applications, vol. 35, no. 1, pp. 87-108, 2006.

[3] A. Bemporad, M. Morari, V. Dua, and E. N. Pistikopoulos, "The explicit linear quadratic regulator for constrained systems," Automatica, vol. 38, no. 1, pp. 3-20, 2002.

[4] A. Grancharova and T. A. Johansen, Explicit Nonlinear Model Predictive Control. Springer, 2012.

[5] B. Grünbaum, Convex polytopes. Wiley Interscience, 1967.

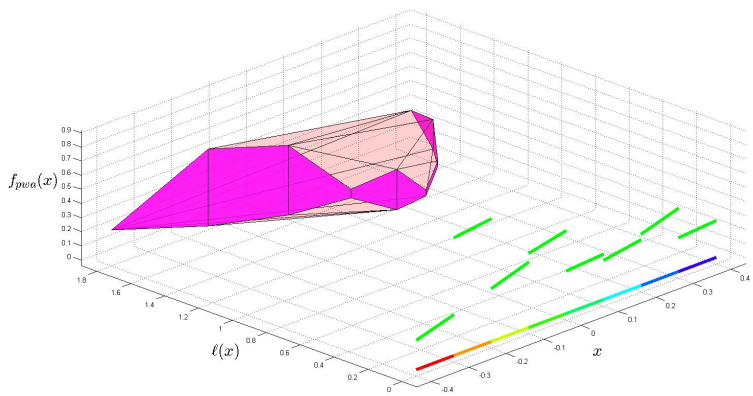

Fig. 4. Illustration for the set of optimal solutions.

[6] M. Gulan, N. Nguyen, S. Olaru, P. Rodriguez-Ayerbe, and B. Rohal'Ilkiv, "Implications of inverse parametric optimization in model predictive control." in Developments in Model-Based Optimization and Control, S. Olaru, A. Grancharova, and F. L. Pereira, Eds. Springer, 2015.

[7] A. B. Hempel, P. J. Goulart, and J. Lygeros, "Every continuous piecewise affine function can be obtained by solving a parametric linear program," in European Control Conference July 17-19, Zurich, Switzerland, 2013.

[8] T. A. Johansen, "On multi-parametric nonlinear programming and explicit nonlinear model predictive control," in Decision and Control, Proceedings of the 41st IEEE Conference on, vol. 3, Dec 2002, pp. 2768-2773.

[9] - "Approximate explicit receding horizon control of constrained nonlinear systems," Automatica, vol. 40, no. 2, pp. 293 - 300, 2004.

[10] M. Kvasnica and M. Fikar, "Clipping-based complexity reduction in explicit mpc," Automatic Control, IEEE Transactions on, vol. 57, no. 7, pp. 1878-1883, 2012.

[11] M. Kvasnica, J. Hledík, I. Rauová, and M. Fikar, "Complexity reduction of explicit model predictive control via separation," Automatica, vol. 49, no. 6, pp. 1776-1781, 2013.

[12] H. N. Nguyen, Constrained Control of Uncertain, Time-Varying, Discrete-Time Systems: An Interpolation-Based Approach. Lecture Notes in Control and Information Sciences, 2014.

[13] N. A. Nguyen, S. Olaru, and P. Rodriguez-Ayerbe, "Inverse parametric linear/quadratic programming problem for continuous pwa functions defined on polyhedral partitions of polyhedra," in 54th IEEE Conference on Decision and Control, Osaka, Japan, 2015.

[14] — " "On the complexity of the convex liftings-based solution to inverse parametric convex programming problems," in European Control Conference, Linz, Austria, 2015.

[15] — - "Recognition of additively weighted voronoi diagrams and weighted delaunay decompositions," in European Control Conference, Linz, Austria, 2015

[16] N. A. Nguyen, S. Olaru, P. Rodriguez-Ayerbe, M. Hovd, and I. Necoara, "Inverse parametric convex programming problems via convex liftings," in 19th IFAC World Congress, Cape Town, South Africa, 2014.

[17] — - "On the lifting problems and their connections with piecewise affine control law design," in European Control Conference, Strasbourg, France, 2014.

[18] S. Olaru and D. Dumur, "A parameterized polyhedra approach for explicit constrained predictive control," in Decision and Control, 43rd IEEE Conference on, vol. 2, 2004, pp. 1580-1585.

[19] E. N. Pistikopoulos, M. C. Georgiadis, and V. Dua, Multi-parametric programming. Wiley-vch, 2007.

[20] E. Pistikopoulos, "Perspectives in multiparametric programming and explicit model predictive control," AIChE journal, vol. 55, no. 8, pp. 1918-1925, 2009.

[21] K. Rybnikov, "Polyhedral partitions and stresses," Ph.D. dissertation, Queen University, Kingston, Ontario, Canada, 1999.

[22] A. Schrijver, Theory of linear and integer programming. John Wiley \& Sons, 1998.

[23] M. M. Seron, G. C. Goodwin, and J. A. Doná, "Characterisation of receding horizon control for constrained linear systems," Asian Journal of Control, vol. 5, no. 2, pp. 271-286, 2003. 